\title{
Low luminosity and ultra low surface brightness galaxies in the Virgo cluster
}

\author{
Nelson Caldwell \\ Smithsonian Astrophysical Observatory, 60 Garden Street, Cambridge, MA, USA \\ email: caldwell@cfa.harvard.edu
}

\begin{abstract}
A CCD survey of selected areas in the Virgo cluster was undertaken with the goal of finding dwarf ellipticals fainter in surface brightness than known in the Local Group. This was successful, and galaxies as much as 2 magnitudes fainter than e.g., Umi, have been found. These tend to be larger in radius, due to the detection methods, and thus more luminous than the LG as well. Two fields containing such galaxies were imaged with ACS/HST, with the result that individual RGB stars have been resolved in these galaxies, which are $15 \mathrm{Mpc}$ distant.
\end{abstract}

Keywords. galaxies: dwarf, galaxies: luminosity function, galaxies: stellar content

\section{Introduction}

What are the limits of dwarf galaxy sizes and densities? Is the star formation history different at the extremes of surface density and luminosity?

These are two questions that I investigated using a CCD survey of several areas within the Virgo cluster. Using the KPNO Mosaic camera on the Mayall 4m telescope, Taft Armandroff \& I took images totaling 6000s in B, and 2900s in R. The combined images of these fields ( 7 in the cluster, 2 outside for background measurements), were then searched for dwarf elliptical galaxies via two techniques:

\subsection{Visual inspection}

Images were displayed on a computer monitor at moderate scale. Galaxies were identified as dEs if they were smooth, and had appropriate sizes and surface brightnesses. At extremely low SB, there is a possibility that the objects are either artifacts, or background clusters of galaxies. The two colors helped both in the sense of requiring multiple detections and appropriate colors (see figure 1). This method is of course tedious and prone to miss obvious objects, but it does find the large, low SB objects which automated techniques tend to miss.

\subsection{Automatic detection}

The same images were analyzed using a combination of SExtractor (Bertin \& Arnouts 1996) and GIM2d (Simard 1998). The former program was used to identify pixels belonging to distinct galaxies (and set the local background), while the latter program was used to fit a model to each galaxy, thus providing structural parameters that can be filtered to exclude objects not conforming to those of a $\mathrm{dE}$ galaxy. This technique is also tedious! But it does allow one to measure the small, low luminosity objects at least in a statistical manner, which the visual technique cannot do.

\section{Virgo Extremes}

Figure 2 shows the resultant distribution of data in the magnitude-surface brightness plane from the automatic method. Objects below the straight line are in the area 


\section{Virgo dE color-magnitude relation}

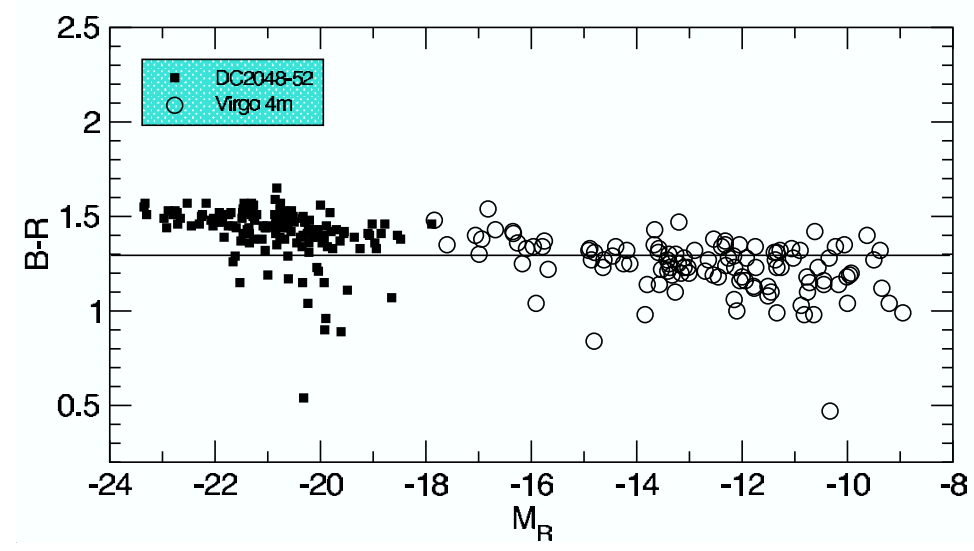

Figure 1. Virgo dE cmd, with brighter galaxies from the cluster DC2048-52 shown for comparison

Galaxies with Sersic $\mathrm{n}<2.5$, smoothness $|\mathrm{s}|<0.1$, ellip $<0.55$

6 Virgo fields and 2 background

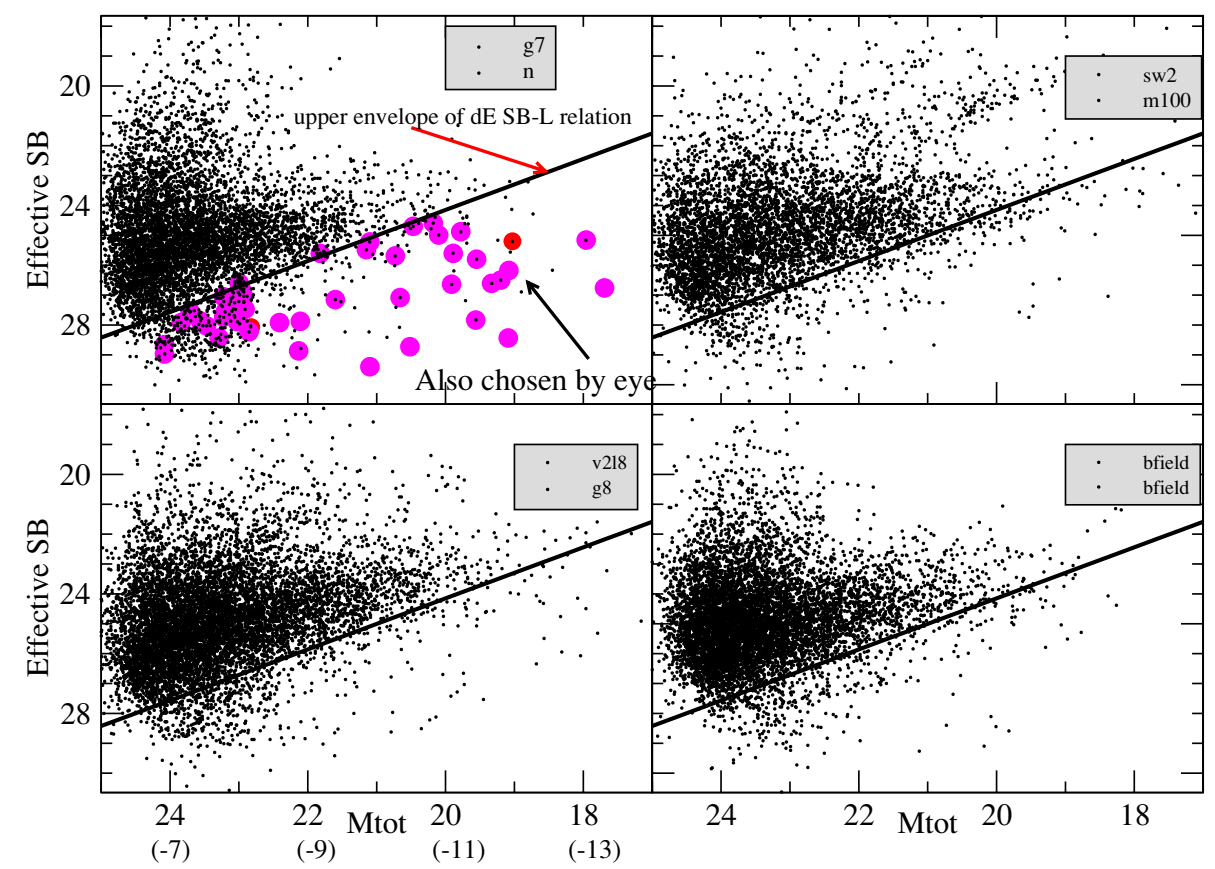

Figure 2. Magnitude-SB relation for Virgo fields

occupied by LG dEs as well as the Virgo dEs found visually. (note that for an exponential profile, effective surface brightness is $1.1 \mathrm{mag}$ fainter than the central surface brightness, independent of scale length).

Two things to note: (1) there are indeed galaxies in the Virgo cluster with B central surface brightnesses down to $27.5 \mathrm{mag} \operatorname{arcsec}^{-2}$, about 2 magnitudes fainter than known 
Surface brightness distribution

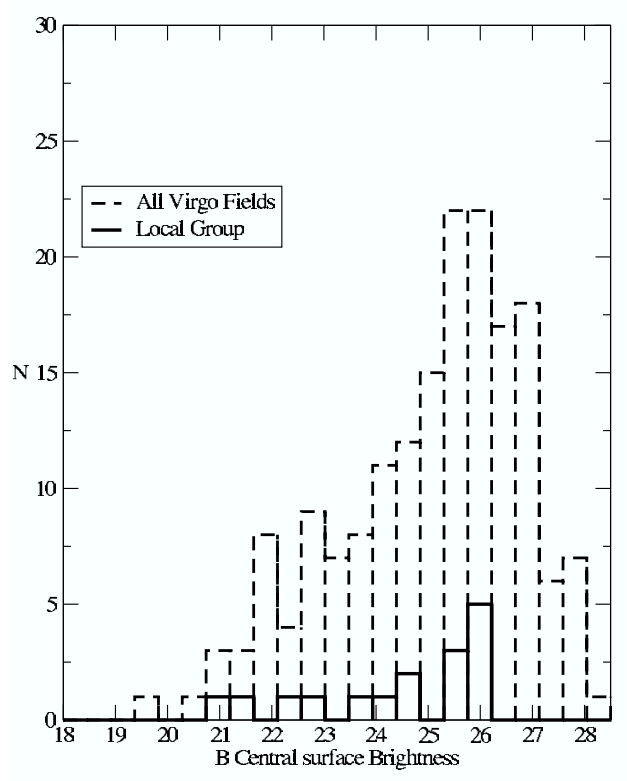

Figure 3. Distribution in surface brightness for Virgo dEs

in the Local Group. The ones I am certain about tend to be larger than the local ones, because the smaller ones could be background objects. Figure 3 shows the distribution of surface brightness in Virgo dwarfs. Clearly, the limit of SB in real objects has still not been reached, despite the low values obtained here.

(2) There are objects in the central parts of Virgo that correspond to the faintest LG dwarfs in luminosity, as indicated by the excess of objects in the lower left corner of the diagram for the Virgo data as opposed to the background fields.

\section{HST/ACS Images}

One might still have a nagging doubt, as did I, whether the extreme galaxies found here are in fact real, or if so, actually in the Virgo cluster. Figure 4 shows two candidates, the one on the left is the most extreme in my catalog, and whose existence was the source of debate even with my collaborator. High resolution imaging is of course the only way to answer these questions, and we were lucky to be allocated time to take a long sequence of exposures on two felds with ACS/HST.

Aside of the ultra low surface brightness galaxy, each field contains 2 other Virgo galaxies as well: one higher surface brightness $\mathrm{dE}$, one extremely low $\mathrm{L} d \mathrm{dE} / \mathrm{d} \operatorname{Irr}\left(\mathrm{M}_{\mathrm{B}}=\right.$ $-8)$, and the halos of an SBab spiral and a luminous $d E\left(\mathrm{M}_{\mathrm{B}}=-15\right)$. Exposure times totaled 33,000s in F555W (V), 14,000s in F814W (I), or 19 orbits per field.

\subsection{Results}

Figure 5 shows cutouts of one of the ACS field. The targeted object is on the left (with the ACS CCD gap running through it). The reproduction may not show it, but we have clearly resolved stars at this location, at the right color and magnitude to identify them as RGB stars in a Virgo galaxy. So this particular galaxy, which has $\mathrm{M}_{\mathrm{B}}=-10.7$ and $\mathrm{B}_{\mathrm{o}}=27.6$, is in fact a member of the Virgo cluster. The central object is one of the 


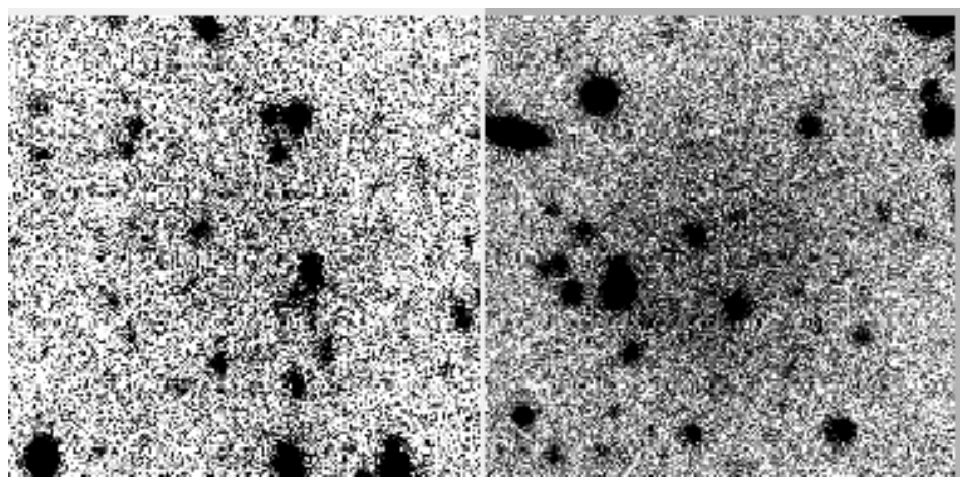

Figure 4. Two Ultra Low LSB targets

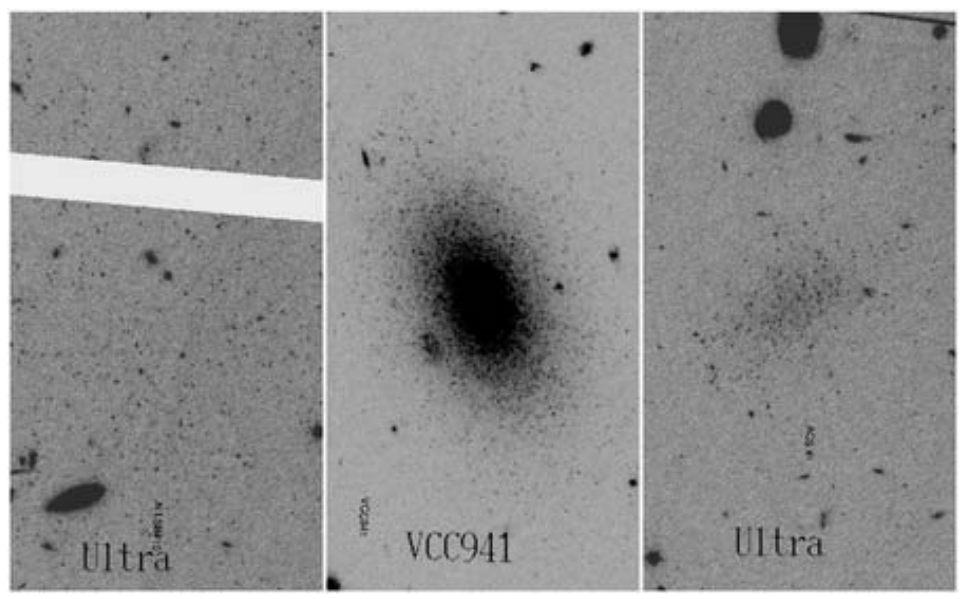

Figure 5. HST/ACS I band images

fainter dE galaxies listed in Binggeli et al. (1985, we thus find $\mathrm{M}_{\mathrm{B}}=-12.1$, but is also bright in surface brightness with $\mathrm{B}_{\mathrm{o}}=23.2$ ). It too is clearly resolved, and is thus also a member. At the right is a bonus galaxy, one picked up by the automated method above, but discounted by me as a background object on inspection. It is in fact also a member, with $\mathrm{M}_{\mathrm{B}}=-8.2$, making it the one of the least luminous galaxies studied.

The second ACS field shows much the same thing: the candidate dE galaxy is wellresolved and thus a cluster member. I am forming stellar color-magnitude diagrams, but difficulties with aperture corrections and zero-points prevents me from publishing them here at this time. In general however, it appears that the metal abundances are low, perhaps $[\mathrm{Fe} / \mathrm{H}] \sim-1.7$, but not extreme. As well, the $\mathrm{dE}$ galaxies do not appear to have large amount of upper AGB stars, so ages are not as young as found in M81-F8D1, for comparison.

\section{Conclusions}

Clearly, extremely low stellar density galaxies do exist, and are even found in the densest regions of the Virgo cluster. One must think that these objects are dark matter dominated, lest they be disrupted easily by the frequent passages of larger galaxies in the core. The large observed range in optical radii among galaxies (a factor of 5) at low 
surface brightness is intriguing. Is this due to star formation processes, or rather DM halo density distribution variations at low mass?

\section{Acknowledgements}

This work has been done in collaboration with Dan McIntosh, T.A. Armandroff, and G.S. Da Costa.

\section{References}

Binggeli, B., Sandage, A. \& Tammann, G. 1985, AJ 90, 1681

Bertin, E. \& Arnouts, S. 1996, A\&AS 117, 393

Simard, L. 1998, ASPC 145, 108

\section{Discussion}

MiEske: If you restrict your sample to those galaxies with $\mu<$ that of the Local Group, is the average size still larger than that of the LG dwarfs?

CALDWELL: If we consider the "candidate" dEs found automatically, via SExtractor and GIM2d, then I think the answer is that the mean Virgo sizes are similar to those in the LG. What is apparent however, is that there are dEs in Virgo with much larger sizes than found in the LG, and currently I don't have a technique to find those automatically in Virgo (they were found by inspection of the images)

Gallagher: Comment: Great project and spectacular results! Question: Can you be sure that these are independent objects and not part of larger debris structures that are common in Virgo (e.g., Mihos CWRU study)?

CALDWELL: The two "ultra-low" surface brightness objects targeted have a definite radial falloff in stellar density from a well-defined center, and, as well as I can measure, are elliptical on the sky. In other words, even with their low central surface brightnesses, they still stand out from their surroundings.

FErguson: Would you have been able to detect AGB stars in your V band images?

CALDwell: Not the reddest ones of course, whose V-I colors would mean that the V magnitudes would be below my detection limit. The behaviour of AGB stars in the M81 dwarf F8D1 though indicates we would expect to see a large number of AGB stars just above the RGB tip, which aren't evident in these Virgo galaxies. 